\title{
IER3IP1 deficiency leads to increased $\beta$-cell death and decreased $\beta$-cell proliferation
}

\author{
Juan Sun ${ }^{1}$ and Decheng Ren ${ }^{1}$ \\ ${ }^{1}$ Department of Medicine, The University of Chicago, Chicago, IL 60637, USA \\ Correspondence to: Decheng Ren, email: decheng@uchicago.edu \\ Keywords: cell death and proliferation, beta-cell, IER3IPI \\ Received: March 06, $2017 \quad$ Accepted: April 27, $2017 \quad$ Published: May 25, 2017 \\ Copyright: Sun et al. This is an open-access article distributed under the terms of the Creative Commons Attribution License \\ 3.0 (CC BY 3.0), which permits unrestricted use, distribution, and reproduction in any medium, provided the original author and \\ source are credited.
}

\section{ABSTRACT}

Mutations in the gene for Immediate Early Response 3 Interacting Protein 1 (IER3IP1) cause permanent neonatal diabetes mellitus in human. The mechanisms involved have not been determined and the role of IER3IP1 in $\beta$-cell survival has not been characterized. In order to determine if there is a molecular link between IER3IP1 deficiency and $\beta$-cell survival and proliferation, we knocked down Ier3ip1 gene expression in mouse MIN6 insulinoma cells. IER3IP1 suppression induced apoptotic cell death which was associated with an increase in Bim and a decrease in Bcl-xL. Knockdown of Bim reduced apoptotic cell death in MIN6 cells induced by IER3IP1 suppression. Overexpression of the anti-apoptotic molecule $\mathrm{Bcl}-\mathrm{xL}$ prevents cell death induced by IER3IP1 suppression. Moreover, IER3IP1 also regulates activation of the unfolded protein response (UPR). IER3IP1 suppression impairs the Inositol Requiring 1 (IRE1) and PKR-like ER kinase (PERK) arms of UPR. The cell proliferation of MIN6 cells was also decreased in IER3IP1 deficient cells. These results suggest that IER3IP1 suppression induces an increase in cell death and a decrease in cell proliferation in MIN6 cells, which may be the mechanism that mutations in IER3IP1 lead to diabetes.

\section{INTRODUCTION}

Immediate early response 3 interacting protein 1 (IER3IP1) is a highly conserved protein in different species and expressed in pancreas, heart, skeletal muscle, fetal brain cortex, kidney, liver, brain, placenta, lung and peripheral blood leukocytes $[1,2]$. Human IER3IPI gene is located on human chromosome 18q12 and has 3 exons encoding an 82-amino acid protein. IER3IP1 contains 2 transmembrane domains and a putative G-patch domain found in several RNA-associated proteins and in type D retroviral polyproteins. IER3IP1 is localized to the endoplasmic reticulum (ER) through its C-terminal transmembrane domain [1]. IER3IP1 may be involved in transporting the proteins between the ER and the Golgi apparatus. Recently, two homozygous missense mutations in IER3IP1 were found in two unrelated consanguineous families [1]. These mutations cause a unique clinical syndrome in which affected individuals display severe infantile epileptic encephalopathy, microcephaly, simplified gyral patterns, and permanent neonatal diabetes mellitus.
Molecular analysis revealed a homozygous missense mutation in exon1 of the Ier3ip1 gene, c.62T $>\mathrm{G}$, causing a valine to glycine amino acid substitution at position 21 (p. Val21Gly), and a second homozygous missense mutation in exon 3, c.233T $>$ C, causing a leucine to proline substitution at position 78 (p.Leu78Pro). These observations were subsequently confirmed by another group [2]. In addition, a novel frame shift mutation p.Phe27fsSer25 and a p.Val21Gly (V21G) mutation in IER3IP1 were found in a neonatal diabetes patient [3]. The association of neonatal diabetes with IER3IP1 mutants suggests that IER3IP1 may regulate $\beta$-cell survival and/ or function.

An increase in $\beta$-cell death and a progressive $\beta$-cell mass reduction as an essential element occur during developing diabetes [4]. ER stress can induce $\beta$-cell death. A number of causes including high glucose, free fatty acids, inflammatory cytokines, hypoxia and mutations in the insulin 2 gene may induce ER stress in $\beta$-cells. ER stress is sensed by three ER transmembrane proteins: Inositol requiring 1 (IRE1), PKR-like ER kinase (PERK), and Activating transcription factor 6 (ATF6). 
Upon activation, these stress sensors transduce a different signaling cascade termed the unfolded protein response (UPR) to recover ER homeostasis [5]. In response to ER stress, IRE1 activates its endoribonuclease activity and splicing of transcription factor X-box protein binding 1 (SXBP1) mRNA by its oligomerization and autophosphorylation. SXBP-1 upregulates chaperone and ER-associated protein degradation (ERAD) protein expression. PERK can phosphorylate the $\alpha$ subunit of eukaryotic initiation factor 2 (eIF $2 \alpha$ ) leading to general protein synthesis inhibition, while increasing translation of activating transcription factor 4 (ATF4), which regulates genes important for ER homeostasis. The translocation of the processed form of ATF6 to the nucleus can upregulate UPR homeostatic effectors involved in protein folding, processing, and degradation [6]. The three arms of the UPR regulate several downstream effectors to reduce ER stress. When ER stress cannot be reduced, the UPR activates death effectors and induces $\beta$-cell apoptosis.

In this study, we will define the role of IER3IP1 in $\beta$-cell survival and proliferation, and identify the mechanism of $\beta$-cell apoptosis induced by IER3IP1 suppression.

\section{RESULTS}

\section{IER3IP1 suppression leads to apoptosis in MIN6 cells}

To investigate whether IER3IP1 regulates $\beta$-cell survival, IER3IP1 shRNA lentivirus was used to knock down (KD) expression of IER3IP1 in MIN6 cells. The mRNA levels of IER3IP1 were significantly reduced to $24 \pm 1 \%$ of control cells on day 4 after infection with lentiviral IER3IP1 shRNA (not shown) and IER3IP1 protein levels were decreased to $22 \pm 2 \%$ of control (Figure 1A). The effect of IER3IP1 KD on cell death was determined using propidium iodide (PI) staining followed by Fluorescence-activated cell sorting (FACS). After infection with IER3IP1 shRNA lentivirus, the proportion of PI staining positive cells increased from $52 \pm 3 \%$ on day 4 to $72 \pm 2 \%$ on day 5 and $86 \pm 2 \%$ on day 6 (all $P<0.001$ ), respectively (Figure 1B). In addition, the cleaved caspase 3 protein levels were increased more than 3-fold in IER3IP1 KD MIN6 cells compared to control cells (Figure 1C). IER3IP1 KD also induced an increase in cleaved poly (ADP-ribose) polymerase (PARP), another apoptosis marker (Figure 1C). The pan-caspase inhibitor Z-VAD significantly inhibited the increase in PI staining positive cells (Figure 1D). The PI staining positive cells were decreased from $60 \pm 2 \%$ in IER3IP1 KD cells to $27 \pm$ $1 \%$ in Z-VAD/IER3IP1 KD cells $(P<0.001)$ (Figure 1D). Immunoblot analysis showed that Z-VAD significantly inhibited IER3IP1 KD-induced caspase3 cleavage (Figure 1E). To further determine the effects of IER3IP1 suppression on cell death, we use terminal dUTP nick end labeling (TUNEL) assay to measure cell apoptosis. The results showed that IER3IP1 KD significantly increased the number of TUNEL positive cells. The TUNEL-labeled cells were inceased from $1.0 \pm 0.2 \%$ in control cells to 31.1 $\pm 2.8 \%$ in IER3IP1 KD cells on day $4(P<0.001)$ (Figure $1 \mathrm{~F})$. Collectively, these findings indicate that reduced IER3IP1 expression induces apoptosis in MIN6 cells.

\section{IER3IP1 suppression decreases mitochondrial membrane potential and increases cytochrome $\mathrm{c}$ release}

During apoptosis, the mitochondrial membrane potential $\left(\Delta \Psi_{\mathrm{m}}\right)$ decreases [7]. To determine the effects of IER3IP1 suppression, mitochondrial membrane potential $\left(\Delta \Psi_{\mathrm{m}}\right)$ was measured by quantifying the average mitochondrial fluorescence intensity of tetramethylrhodamine, ethyl ester (TMRE). TMRE uptake into mitochondria was dramatically decreased in IER3IP1 KD cells, indicating that IER3IP1 KD significantly decreased mitochondrial membrane potential (Figure 2A). The relative membrane potential fluorescence in mitochondria decreased by $60 \%$ in IER3IP1 KD cells compared to control cells $(P<0.001)$ (Figure $2 \mathrm{~B})$. IER3IP1 KD induced an increase in cytochrome $\mathrm{c}$ release from mitochondria, the key event in activating apoptosis (Figure 2C). Cytochrome c release increased from $6.5 \pm$ $0.4 \%$ in control cells to $34.4 \pm 4.3 \%$ in IER3IP1 KD cells $(P<0.001)$ (Figure 2D).

\section{IER3IP1 suppression activates Bax in MIN6 cells}

IER3IP1 KD did not induce a significant increase in Bax and Bak mRNA levels in MIN6 cells (not shown). Western blotting also showed no increase in Bax and Bak protein levels in IER3IP1 KD cells compared to control cells (Figure 3A). Since Bax is stationed in the cytosol and can translocate into mitochondria once activated by a diversity of stress. The Bax conformational change stimulated by stress was examined using the monoclonal antibody 6A7, which only recognizes the N-terminal epitope of Bax [8]. The results showed that the amount of Bax precipitated by $6 \mathrm{~A} 7$ anti-Bax antibody was increased in IER3IP1 KD cells (Figure 3B). Bax translocation to mitochondria induced by IER3IP1 KD was also increased (Figure 3C). Bax KD inhibited the increase of Bax translocation to mitochondria (Figure 3C). The PI staining positive cells (cell death) significantly decreased from 68.0 $\pm 1.7 \%$ in IER3IP1 KD cells to $41.8 \pm 4.3 \%$ in IER3IP1/ Bax DKD cells $(P<0.001)$ (Figure 3D).

\section{Bim is increased following IER3IP1 suppression and contributes to cell apoptosis induced by IER3IP1 suppression}

Since Bcl-2 family members are widely involved in apoptotic cell death, the mRNA levels of pro-apoptotic 
Bcl-2 family members were determined by real-time quantitative PCR. Indeed, IER3IP1 KD resulted in an increase in Bim mRNA levels by $70 \%(\mathrm{P}<0.001)$ (Figure 4A). Western blotting showed a corresponding increase in Bim protein levels by $100 \%(P<0.01)$ and a 2.5 - fold increase in cleaved caspase 3 protein $(P<0.001)$, respectively. However, Puma protein levels were unchanged in IER3IP1 KD cells (Figure 4B).

To determine whether Bim up-regulation is the key factor for cell death induced by IER3IP1 suppression, we knock down Bim by the lentiviral shRNA in MIN6 cells. The results showed that Bim protein levels were significantly decreased in Bim KD cells compared to control cells (Figure 4C). The increase in cleaved caspase 3 was also inhibited in Bim/IER3IP1 double knockdown (DKD) cells compared to IER3IP1 KD cells alone (Figure 4C). Following IER3IP1 knockdown, $72.4 \pm 4.9 \%$ of the MIN6 cells took up the PI stain, while in the Bim/IER3IP1 DKD group, only $40.5 \pm 2.5 \%(P<0.001$ compared to IER3IP1 alone) took up the PI stain indicative of a $32 \%$ decrease in dead cells (Figure 4D). Since Bim is able to be regulated at the transcriptional level by FoxO1 and FoxO3a and the post-translational level by Erk, we determined whether FoxO1, FoxO3a and Erk are involved in Bim upregulation induced by IER3IP1 KD. Unexpectedly, both FoxO1 and FoxO3a were decreased in
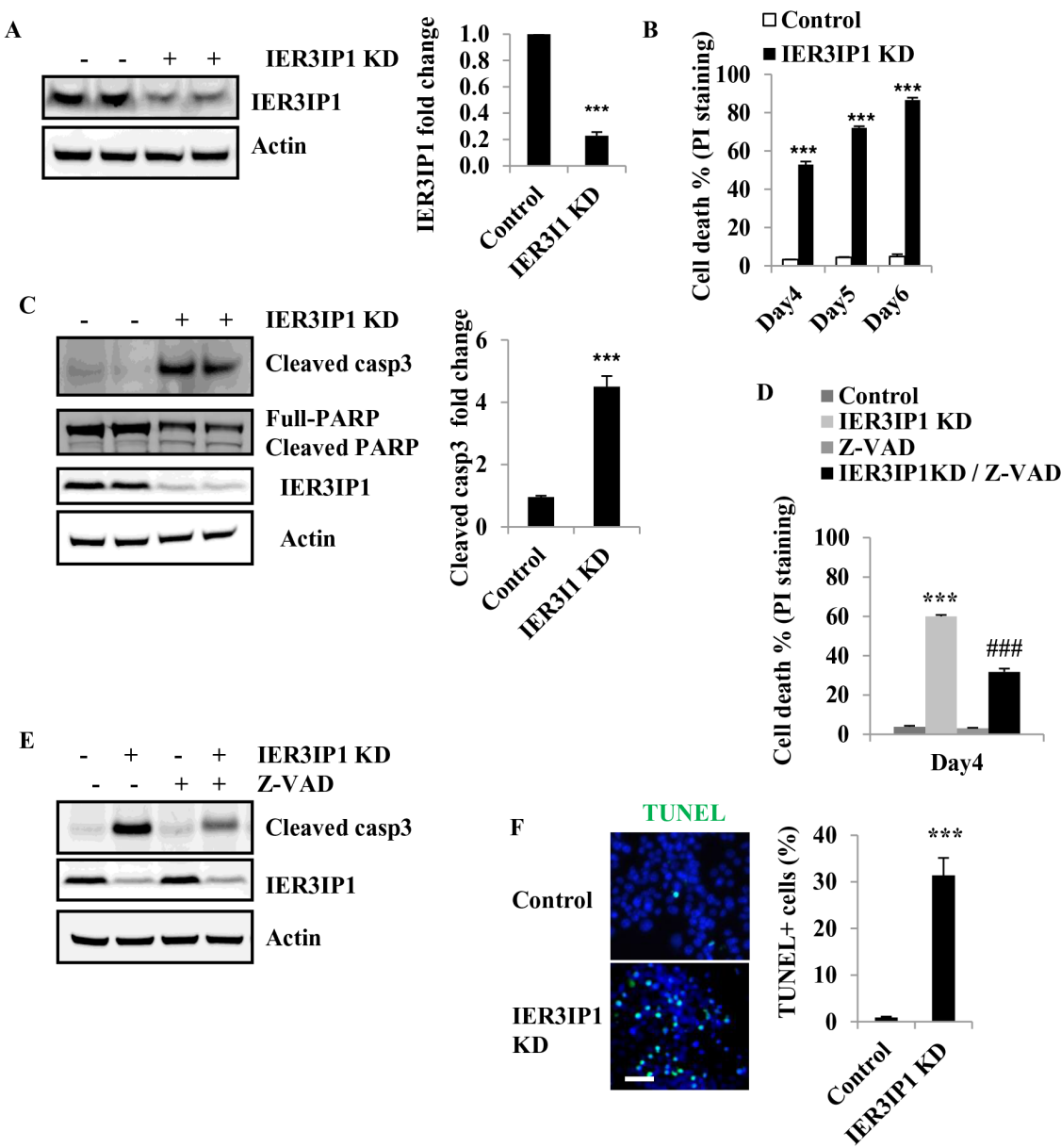

Figure 1: Reduced IER3IP1 expression leads to apoptosis in MIN6 cells. (A) IER3IP1 protein levels in IER3IP1 KD MIN6 cells. MIN6 cells were infected with a lentivirus vector that drives expression of shRNA that targets the IER3IP1 transcript (IER3IP1 KD) or control lentivirus vector that does not have a specific target. IER3IP1 protein levels were measured 4 days after infection by western blot $(\mathrm{n}=3)$. The loaded proteins in the two "- or +" lines were the same but from the different plates. (B) Cell death was determined by PI-staining in MIN6 cells after infection with IER3IP1 shRNA lentivirus. (C) Cleaved caspase3 protein levels in IER3IP1 KD cells. 4 days after infection with IER3IP1 shRNA lentivirus, cleaved caspase 3 and PARP proteins were assayed by western blot ( $\mathrm{n}=3$ ). The loaded proteins in the two "- or +" lines were the same but from the different plates. (D) Z-VAD inhibits caspase3 cleavage induced by IER3IP1 KD. MIN6 cells were treated with the caspase inhibitor Z-VAD $(20 \mu \mathrm{M})$, for 2 hours prior to infection with IER3IP1 shRNA lentivirus. 4 days later, cleaved caspase 3 protein was assayed by western blot $(n=3)$. (E) Z-VAD inhibits cell death induced by IER3IP1 KD in MIN6 cells. MIN6 cells were treated with $20 \mu \mathrm{M}$ Z-VAD, for 2 hours prior to infection with IER3IP1 shRNA lentivirus and cell death was determined by PI-staining on day $4(\mathrm{n}=3)$. $* * * \mathrm{P}<0.001$ compared to control group. \#\#\#P $<0.001$ compared to IER3IP1 KD group. (F) TUNEL labeling of IER3IP1 KD MIN6 cells. 4 days after infection with IER3IP1 shRNA lentivirus, apoptotic cells were assayed by TUNEL staining. Quantitative TUNEL data are shown. Scale bar, $20 \mu \mathrm{m} . * * * \mathrm{P}<0.001$ compared to control group. Values are mean $\pm \mathrm{SEM}$. 
IER3IP1 KD cells compared to control cells (Figure 4E). Moreover, phosphorylation of Erk were also decreased in IER3IP1 KD cells (Figure 4F). The results indicate that Bim upregulation in IER3IP1 KD cells is not mediated by an increase in the expression of FoxO1, FoxO3a and Erk.

\section{Bcl-xL prevents Bim increase and reduces cell death in IER3IP1 KD MIN6 cells}

To determine whether IER3IP1 KD can also induce changes in anti-apoptotic Bcl-2 family members, the protein levels of Bcl-2, Bcl-xL and $\mathrm{Mcl}-1$ were determined. The results showed that Bcl-xL protein levels were decreased in IER3IP1 KD cells. IER3IP1 $\mathrm{KD}$ did not change the protein levels of Bcl-2 and Mcl1 (Figure 5A). To determine whether increasing Bcl-xL expression can inhibit IER3IP1 KD-induced cell death,
Bcl-xL was overexpressed in MIN6 cells. The results showed that Bcl-xL inhibited the increase in cleaved caspase 3 protein induced by IER3IP1 suppression (Figure $5 \mathrm{~B})$. Overexpression (OE) of Bcl-xL also decreased the enhanced cell death in IER3IP1 KD cells. Following overexpression of $\mathrm{Bcl}-\mathrm{xL}$, the cells staining positive for $\mathrm{PI}$ decreased from $90.4 \pm 0.9 \%$ in IER3IP1 KD cells to 21.6 $\pm 0.8 \%(P<0.001)$ in IER3IP1 KD/Bcl-xL OE cells on day 6 (Figure 5C). Taken together, these findings demonstrate that the increase of Bim induces cell death in IER3IP1 KD MIN6 cells and this effect is inhibited by Bcl-xL OE.

\section{IER3IP1 suppression decreases UPR activation}

Since IER3IP1 protein is present in the ER, we determined whether IER3IP1 suppression can cause ER stress and induce the UPR. Surprisingly, mRNA levels

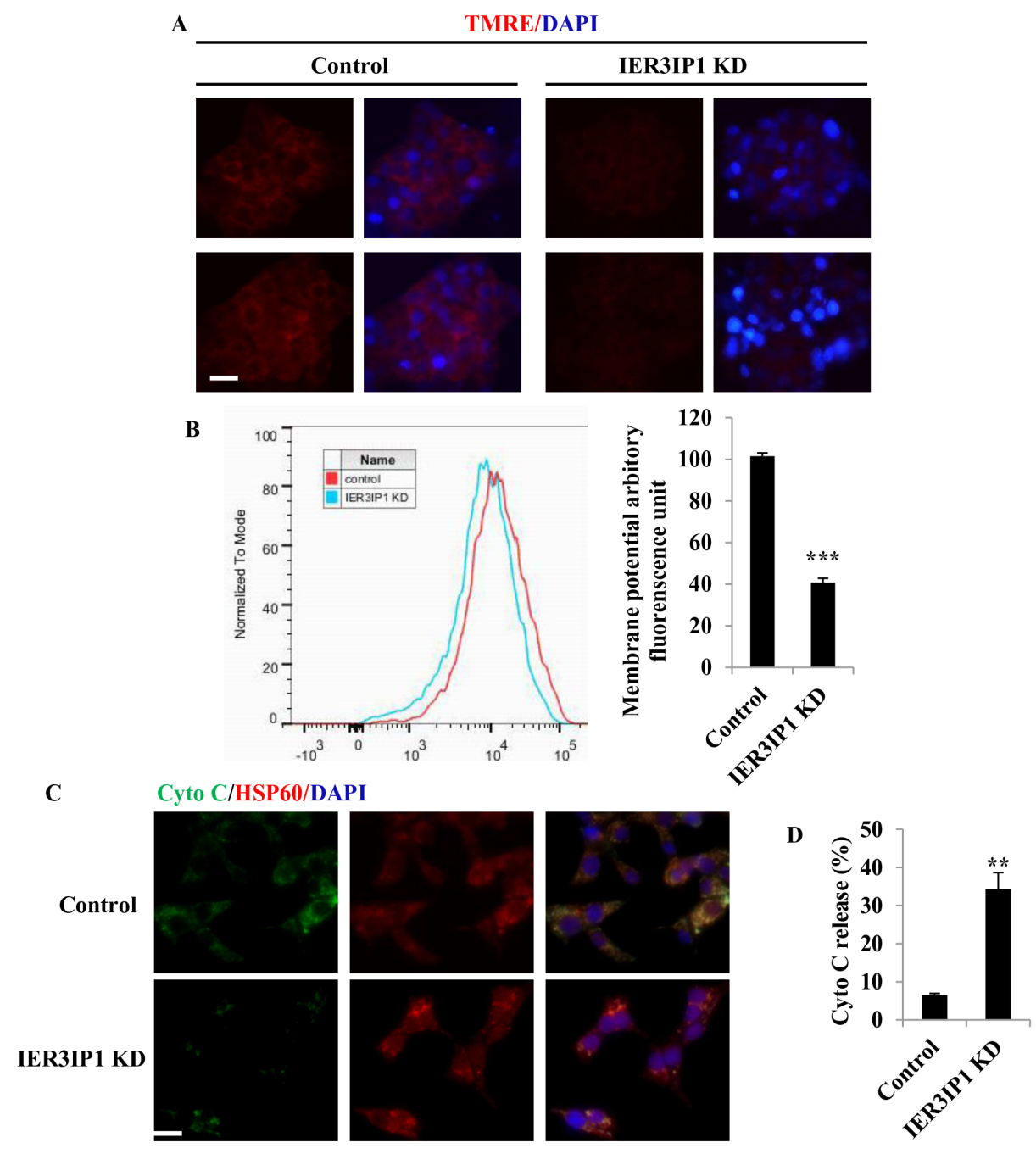

Figure 2: Effects of IER3IP1 suppression on $\Delta \Psi \mathrm{m}$ and cytochrome c release. $(\mathrm{A}+\mathrm{B}) \mathrm{MIN6}$ cells were treated with IER3IP1 shRNA lentivirus for 3 days. Cells were then stained with TMRE dye to measure $\Delta \Psi_{\mathrm{m}}$. Scale bar, $20 \mu \mathrm{m}$. $* * * \mathrm{P}<0.001$. (C+D) IER3IP1 suppression induces cytochrome c release. 3 days after IER3IP1 KD in MIN6 cells, the images were taken under fluorescence microscopy (C). The green, red and blue staining represent cytochrome c, HSP60 and DAPI, respectively. Scale bar, $20 \mu \mathrm{m}$. The release of cytochrome c was also measured (D). ${ }^{* *} \mathrm{P}<0.01$ compared to control group. Values are mean $\pm \mathrm{SEM}$. 
of IRE1 $\alpha$, Perk and ATF6, were significantly decreased in IER3IP1 KD cells compared to control cells $(\mathrm{P}<0.01$ or $\mathrm{P}<0.05$ ) (Figure 6A) indicating that UPR activation is decreased in IER3IP1 KD cells. To further confirm the role of IER3IP1 in UPR activation, the levels of UPR signaling proteins were measured. Both phosphorylation and protein levels of IRE1 $\alpha$ were dramatically decreased (Figure 6B). The expression of spliced XBP-1, a downstream target of IRE1 $\alpha$, was reduced with IER3IP1 KD (Figure 6B). Moreover, mRNA levels of downstream effectors of sXBP-1 including Erdj4, p58IPK, EDEM and Sec61a, were all significantly decreased $(\mathrm{P}<0.01$ or $\mathrm{P}<0.05$ ) (Figure 6C). In addition, although protein levels of PERK were unchanged, phosphorylation of PERK was dramatically decreased (Figure 6D). Phosphorylation levels of eIF2a and protein levels of ATF4, two downstream targets of PERK, were decreased (Figure 6D). Unfortunately, ATF6 proteins were undetectable using the commercial antibodies in MIN6 cells. To further determine the effect of IER3IP1 suppression on UPR activation, the expression of spliced XBP-1 protein induced by ER stressors TC and TG was measured in IER3IP1 KD MIN6 cells. Tunicamycin (TC, induces ER stress by inhibiting

$\mathbf{A}$
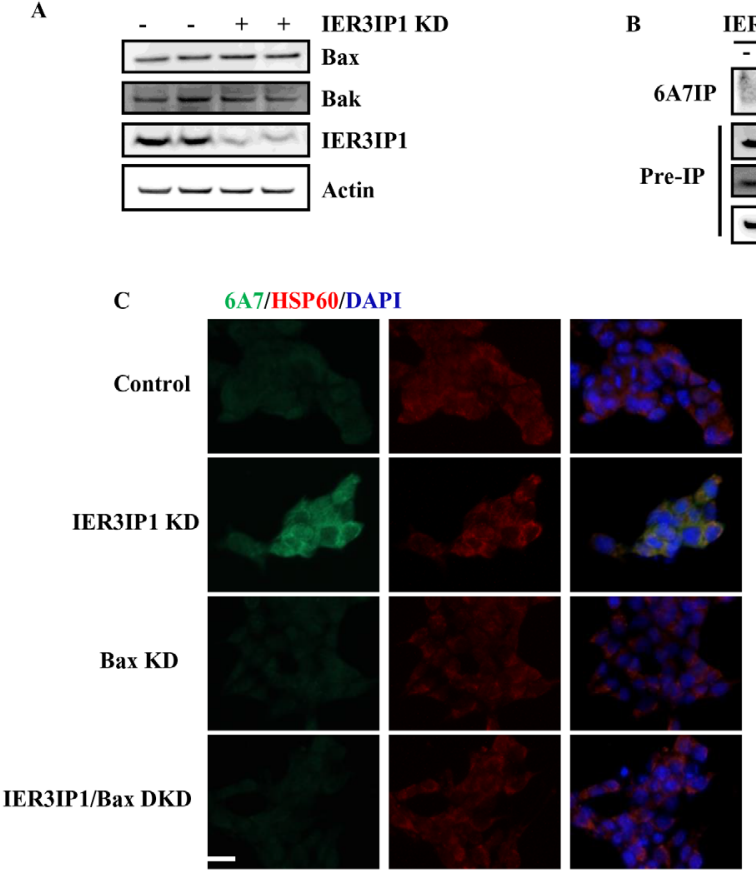

$\mathrm{N}$-linked glycosylation) and thapsigargin (TG, induces ER stress by inhibiting sarco/endoplasmic reticulum calcium ATPase) significantly increased spliced XBP-1 and Chop in control cells (Figure 6E). However, compared to control groups, IER3IP1 KD significantly inhibited the increase in protein levels of sXBP-1 and Chop induced by TC and TG treatment (Figure 6E). These data suggest that IER3IP1 decreases UPR activation at least through shutting down the IRE $1 \alpha$ and PERK arms of the UPR.

\section{IER3IP1 suppression decreases cell proliferation in MIN6 cells}

Besides cell survival, we also determine whether IER3IP1 regulates cell proliferation. The proliferative marker Ki67 staining showed that proliferation of cells was decreased by $42 \%$ in IER3IP1 KD cells compared with control cells (Figure 7A). To elucidate the mechanism by which IER3IP1 modulates cell proliferation, we analyzed the proteins related to cell proliferation including protein kinase B (PKB, AKT), 40S ribosomal protein S6 (S6) and eukaryotic initiation factor 4E (eIF4E) binding protein (4E-BP) $[9,10]$. Phosphorylation levels of Akt at Ser-473
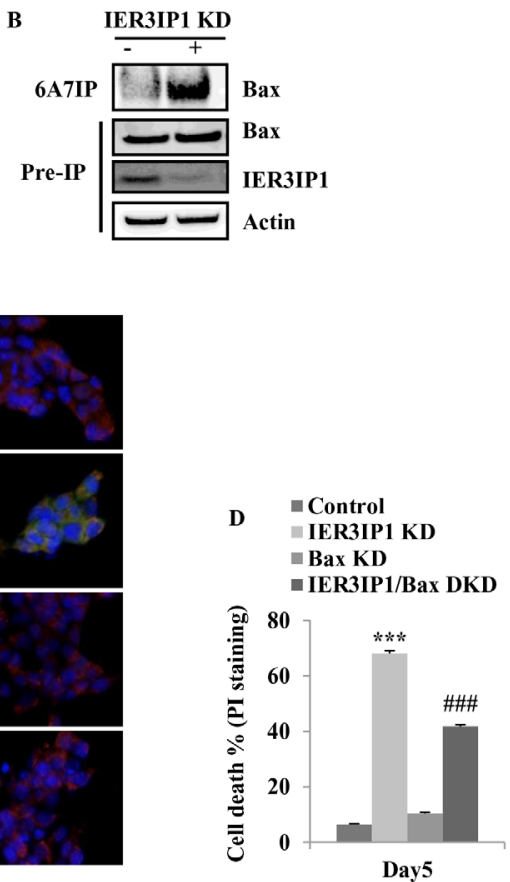

Figure 3: IER3IP1 suppression induces Bax activation in MIN6 cells. (A) Bax and Bak protein levels in control and IER3IP1 KD cells. 3 days after IER3IP1 KD in MIN6 cells, Bax and Bak protein levels showed no difference between control and IER3IP1 KD cells $(n=3)$. The loaded proteins in the two "- or +" lines were the same but from the different plates. (B) Immunoprecipitate of Bax. 3 days after IER3IP1 KD in MIN6 cells, cells were lysed in 1\% CHAPS and then immunoprecipitated with the 6A7 anti-BAX antibody. Immunoprecipitates were analyzed by anti-BAX (N20) immunoblots. (C) Bax KD inhibits IER3IP1 KD-induced Bax activation. 3 days after IER3IP1 KD in MIN6 cells, the images were taken under fluorescence microscopy. Scale bar, $20 \mu \mathrm{m}$. (D) Bax KD inhibits cell death induced by IER3IP1 suppression. MIN6 cells were infected with IER3IP1 shRNA or IER3IP1/Bax double shRNA lentivirus. 4 days later,

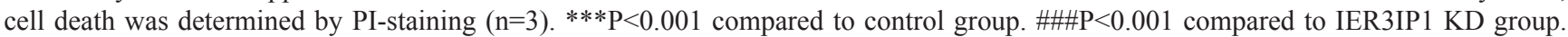
Values are mean \pm SEM. 
(p-AKT S473) and Thr-308 (p-AKT T308) were decreased in IER3IP1 KD cells (Figure 7B). IER3IP1 suppression also induced a decrease in protein levels of p-4E-BP and p-S6 (Figure 7C). Taking the results together, we can conclude that both AKT and mTOR signaling pathways are involved in IER3IP1-regulated cell proliferation. Our working model suggests that IER3IP1 suppression induces an increase in cell death and a decrease in cell proliferation in MIN6 cells (Figure 7D).

\section{DISCUSSION}

IER3IP1 is thought to be involved in the endoplasmic reticulum stress response [1]. Clinical studies have demonstrated that neonates with mutations in IER3IP1 develop early-onset permanent diabetes $[1,2]$. An autopsy specimen from one patient showed increased apoptosis in pancreatic $\beta$-cells indicating that $\beta$-cell death may be the pathogenetic mechanism for the neonatal diabetes in this syndrome [1]. However, the role of IER3IP1 in $\beta$-cells and how IER3IP1 deficiency causes $\beta$-cell death are unclear. The present studies were undertaken to define the molecular mechanisms responsible for cell death induced by IER3IP1 suppression. The results demonstrate that IER3IP1 suppression in MIN6 cells induces apoptotic cell death detected by caspase 3 cleavage, increased TUNEL labeling and Bim upregulation. Bim is a $\mathrm{BH} 3$-only molecule that is essential for mitochondrial-dependent apoptosis and for inducing cell death in different cell types including neurons, $\mathrm{T}$ and B lymphocytes and macrophages [11-15]. Our recent studies have demonstrated that Bim is increased in Pdx1 deficient $\beta$-cells, and Bim, Puma and Bax are required for $\beta$-cell apoptosis induced by Pdx1 deficiency [16,

\section{A}

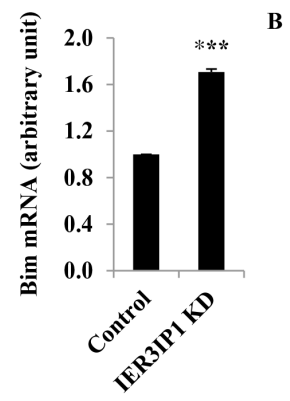

C

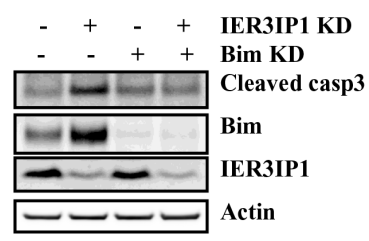

$\mathbf{E}$

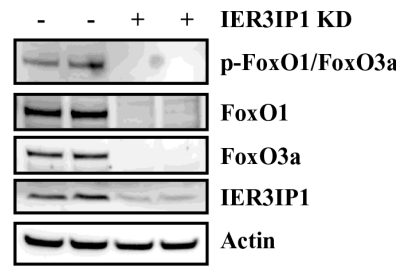

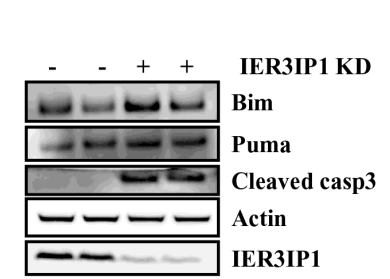

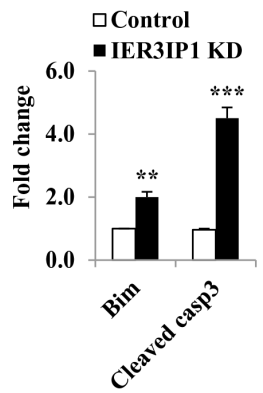

D

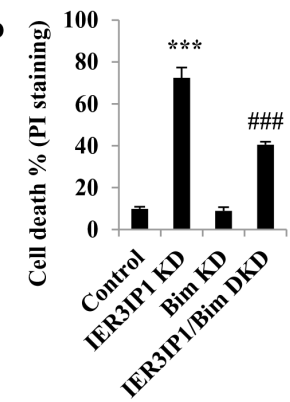

F

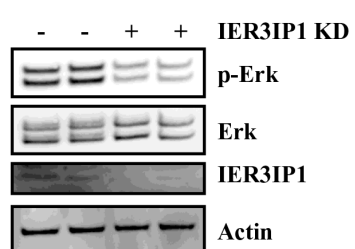

Figure 4: Bim is increased in MIN6 cells following IER3IP1 suppression. (A) Bim mRNA levels in control and IER3IP1 KD cells. 4 days after IER3IP1 KD in MIN6 cells, Bim mRNA levels were measured by real time quantitative reverse transcription-PCR (qRT-PCR) in MIN6 cells ( $\mathrm{n}=3)$. $* * * \mathrm{P}<0.001$ compared to control group. Values are mean $\pm \mathrm{SEM}$. (B) Bim and Puma protein levels in IER3IP1 KD cells. 4 days after IER3IP1 KD in MIN6 cells, immunoblot analysis was performed to determine Bim, Puma and cleaved caspase3 protein levels in IER3IP1 KD MIN6 cells. The bar graph depicts the relative changes in the levels of the indicated proteins using densitometry analysis of the Western blots $(n=3)$. Values are mean \pm SEM. The loaded proteins in the two "- or +" lines were the same but from the different plates. (C) Western blot of IER3IP1/Bim DKD cells. 4 days after IER3IP1/Bim DKD in MIN6 cells, immunoblot of Bim and cleaved caspase 3 in cells. (D) Measurement of cell death. 4 days after Bim/IER3IP1 DKD in MIN6 cells, cell death was determined by PI-staining ( $\mathrm{n}=3)$. ${ }^{* * *} \mathrm{P}<0.001$ compared to control group. \#\#\#P<0.001 compared to IER3IP1 KD group. Values are mean $\pm \mathrm{SEM}$. (E+F) Western blot of IER3IP1 KD MIN6 cells. 4 days after IER3IP1 KD in MIN6 cells, phosphorylation of FoxO1/FoxO3a, FoxO1, FoxO3a (E), p-Erk and Erk (F) were determined by Western blot. The loaded proteins in the two "- or +" lines were the same but from the different plates. 
17]. The present experiments demonstrated that Bim KD significantly reduced IER3IP1 suppression-induced cell death. Furthermore, we demonstrated that IER3IP1 suppression induces Bax activation. Our previous studies showed that Bim can directly activate Bax and induce Bax translocation to the mitochondria leading to cell apoptosis $[8,18]$. Cells survive or undergo death is decided by the balance between pro-apoptotic and anti-apoptotic factors. In IER3IP1 KD MIN6 cells, the anti-apoptotic molecule Bcl-xL was significantly decreased while Mcl-1 and Bcl2 were unchanged. Overexpression of the anti-apoptotic molecule Bcl-xL can prevent cell death induced by IER3IP1KD. Since Bcl-xL can bind to Bim and sequester it, the decrease of $\mathrm{Bcl}-\mathrm{xL}$ causes Bim release from $\mathrm{Bcl}-$ $\mathrm{xL}$ and mediates $\beta$-cell apoptosis in IER3IP1 KD cells. The mechanism by which IER3IP1 suppression induces the reduction of $\mathrm{Bcl}-\mathrm{xL}$ needs to be further investigated.

Bim is expressed in the acinar cells, endocrine cells of the islets and the columnar epithelial cells in mouse. Bim is also expressed in $\beta$-cells of human islets
[19]. Misregulation of Bim in $\beta$-cells is related to type 1 and 2 diabetes $[20,21]$. Bim activity can be regulated through transcriptional and posttranslational mechanisms. Forkhead transcription factors can increase Bim transcription $[22,23]$. As for posttranslational regulation, Bim can be phosphorylated by Erk1/2 (extracellular signal-regulated kinase $1 / 2$ ) resulting in its ubiquitylation, proteasomal targeting and degradation [24, 25]. For example, transcriptional regulation of Bim by FoxO3a mediates hepatocyte lipoapoptosis [22]. To determine whether FoxO1 or FoxO3a mediates Bim upregulation induced by IER3IP1 suppression, the protein levels of FoxO1or FoxO3a were determined in IER3IP1 KD cells. Our results showed that both FoxO1 and FoxO3a were decreased in IER3IP1 KD cells, suggesting that mRNA upregulation of $\mathrm{Bim}$ is not mediated by FoxO1 and FoxO3a. Our data further showed that Erk is also not involved in regulating Bim expression. Since IER3IP1 is located in the ER and ER plays a key role in maintaining calcium homeostasis, IER3IP1 suppression may interrupt
A
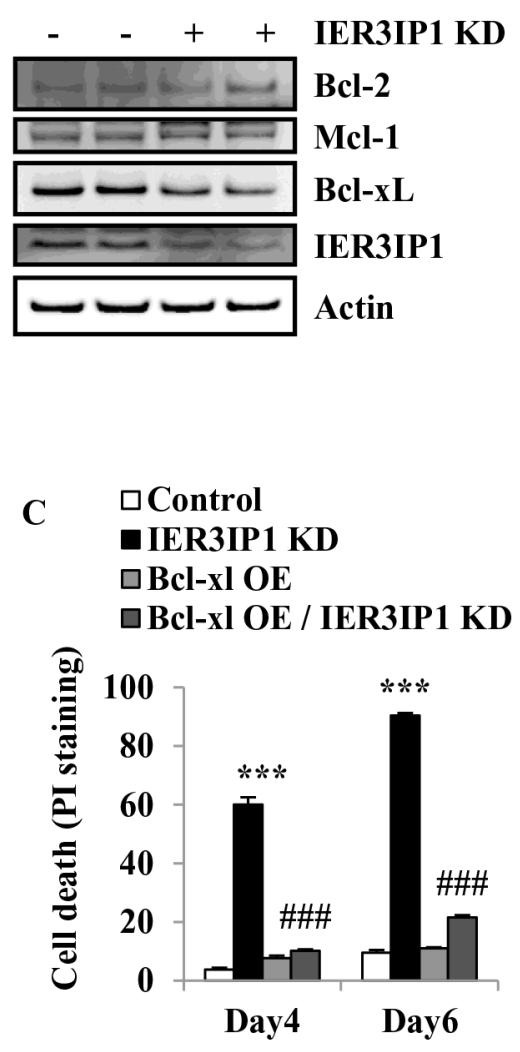

B

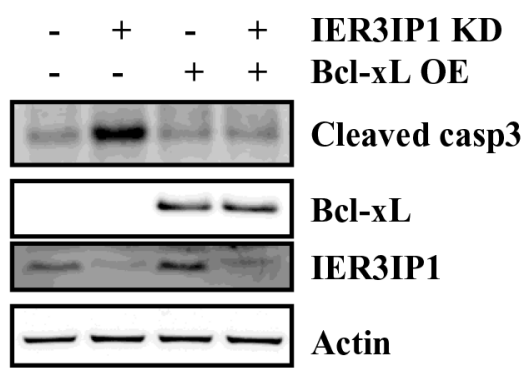

Figure 5: Overexpression of Bcl-xL reduces cell death induced by IER3IP1 suppression. (A) Protein levels of anti-apoptotic Bcl-2 family members in IER3IP1 KD cells. 4 days after infection with IER3IP1 shRNA lentivirus, protein levels of Bcl-2, Bcl-xL and Mcl-1 in MIN6 cells were analyzed by Western blot. The loaded proteins in the two "- or +" lines were the same but from the different plates. (B) Effect of overexpression of Bcl-xL on the cleavage of caspase3. MIN6 cells were infected with IER3IP1 shRNA lentivirus and retrovirus overexpressing Bcl-xL for 3 days, then protein levels of cleaved caspase 3 and Bcl-xL were determined by Western blot. (C) Cell death was determined by PI-staining. MIN6 cells were infected with IER3IP1 shRNA lentivirus and Bcl-xL-overexpressed retrovirus, cell death was determined by PI-staining on day 4 and day $6(\mathrm{n}=3)$. ${ }^{* * *} \mathrm{P}<0.001$ compared to control group. \#\#\#P<0.001 compared to IER3IP1 $\mathrm{KD}$ group. Values are mean $\pm \mathrm{SEM}$. 
calcium homeostasis. Studies showed that calcium could regulate Bim-mediated cell death and Bim-deficient retinal explants are resistant to calcium overload [26]. Thus whether calcium regulates Bim and the mechanism by which Bim is upregulated need to be further explored.

Multiple factors can disturb ER homeostasis and cause accumulation of unfolded proteins, which will eventually trigger the UPR [27]. The initial effect of the UPR is to reestablish homeostasis and recover ER function by activation of transcriptional programs to induce expression of genes that are capable of enhancing the protein folding capacity of the ER and genes for ERAD. When the UPR fails to compensate, ER stress is not mitigated and homeostasis is not restored, cell death is induced, typically by apoptosis. In this study, at least two arms of UPR, including IER $1 \alpha-$ XBP-1, and PERK-eIF2a and -ATF4, are decreased in IER3IP1 KD cells. Furthermore, even in MIN6 cells treated with ER stressors, IER3IP1 suppression shuts down activation of the UPR. The loss of PERK expression in humans and mice increases $\beta$-cell apoptosis due to a failure of UPR regulation [28, 29]. IRE1 $\alpha$-sXBP-1 signaling pathway also appears to be important for regulating insulin biosynthesis, $\beta$-cell survival and proliferation [30]. The transcriptional targets of sXBP1 are highly enriched for ERAD factors, ER chaperones, and enzymes [31].
A

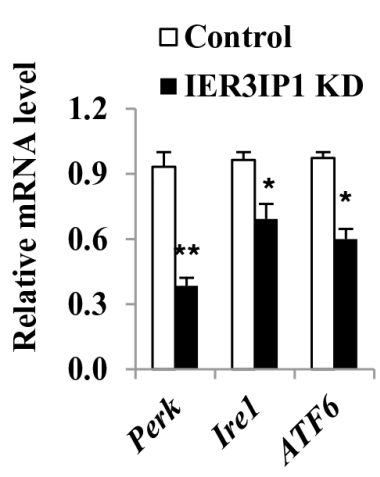

B

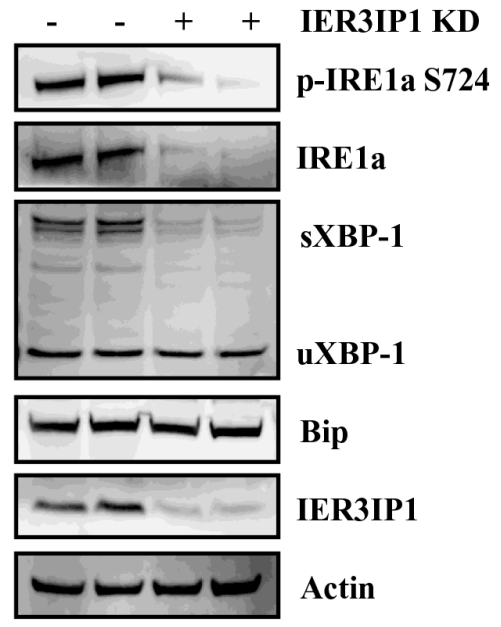

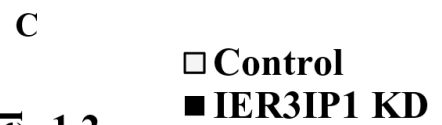

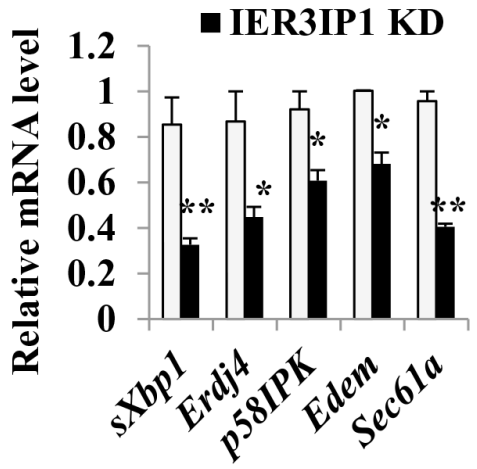

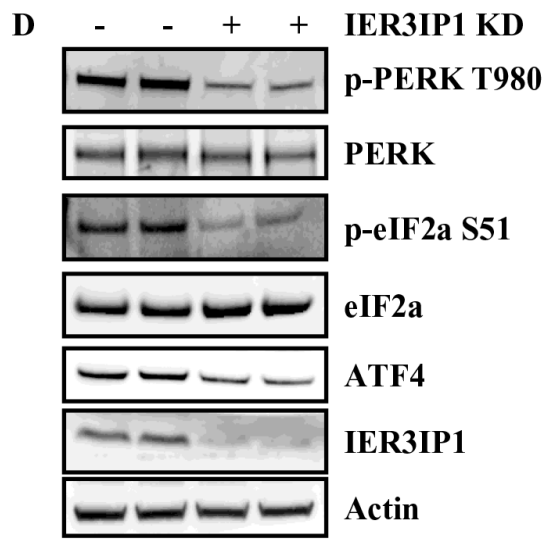

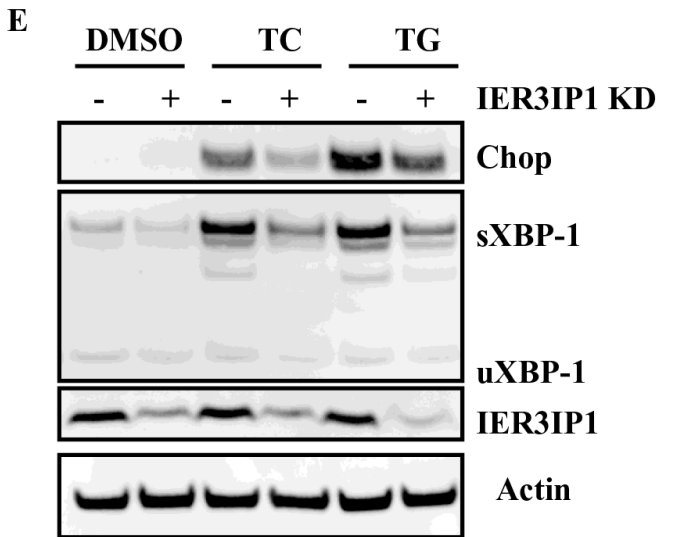

Figure 6: IER3IP1 suppression decreases the UPR activation. (A) mRNA levels of IRE1 $\alpha$, Perk and Atf6 in IER3IP1 KD cells. 4 days after infection with IER3IP1 shRNA lentivirus, mRNA levels of IRE1 $\alpha$, Perk and Atf6 in IER3IP1 KD cells were determined by qRTPCR. ${ }^{*} \mathrm{P}<0.05,{ }^{*} \mathrm{P}<0.01$ compared to control group. Values are mean $\pm \mathrm{SEM}$. (B) Effect of IER3IP1 suppression on the IRE1 $\alpha$ arm of the UPR. 4 days after IER3IP1 KD in MIN6 cells, phosphorylation of IRE1 $\alpha$, Bip, sXBP-1 and uXBP-were determined by Western blot. The loaded proteins in the two "- or +" lines were the same but from the different plates. (C) mRNA levels of downstream targets of sXBP-1 in IER3IP1 KD cells. 4 days after IER3IP1 KD in MIN6 cells, mRNA levels of downstream targets of sXBP-1, Erdj4, p58IPK, EDEM and Sec61a, were determined by qRT-PCR. Values are mean \pm SEM. (D) Effect of IER3IP1 suppression on the Perk arm of the UPR. 4 days after IER3IP1 KD in MIN6 cells, phosphorylation of Perk and eIF2a, and ATF4 were determined by Western blot. The loaded proteins in the two "- or +" lines were the same but from the different plates. (E) IER3IP1 KD decreases UPR activation induced by ER stress in MIN6. 4 days after IER3IP1 KD, MIN6 cells were treated with $10 \mu \mathrm{g} / \mathrm{ml}$ tunicamycin (TC) or $1 \mu \mathrm{M}$ thapsigargin (TG) for 6 hours. Then the expression levels of Chop, sXBP-1, uXBP-1 and IER3IP1 were determined by Western blot. 
The decrease in SXBP-1 in IER3IP1 KD cells therefore reduces the ER's capacity for ERAD. The two arms of UPR defect in IER3IP1 KD cells lead to the increase in ER stress and cell death. Furthermore, ER is an important organ to maintain $\mathrm{Ca}^{2+}$ homeostasis. IER3IP1 deficiency in ER may disrupt $\mathrm{Ca}^{2+}$ homeostasis. Cellular $\mathrm{Ca}^{2+}$ overload or $\mathrm{Ca}^{2+}$ leakage from the cells can cause cell death [32]. However, the mechanism by which IRE3IP1 maintains ER homeostasis is unclear and whether IER3IP1 regulates $\mathrm{Ca}^{2+}$ homeostasis need to be investigated. In addition, ATF4 and Chop can regulate Bim expression, however, in this study both ATF4 and Chop decreased, which suggests that Bim expression is also ATF4- and Chop- independent. Whether there is a crosstalk between UPR inactivation and Bim upregulation or a decrease of Bcl-xL needs to be further studied.

Since PI3K/AKT/mTOR was the most relevant pathway relating to $\beta$-cell proliferation, survival, and cell growth $[33,34]$, the effects of IER3IP1 KD on AKT/ mTOR signaling pathways were determined in this study. We observed a significant decrease in protein levels of

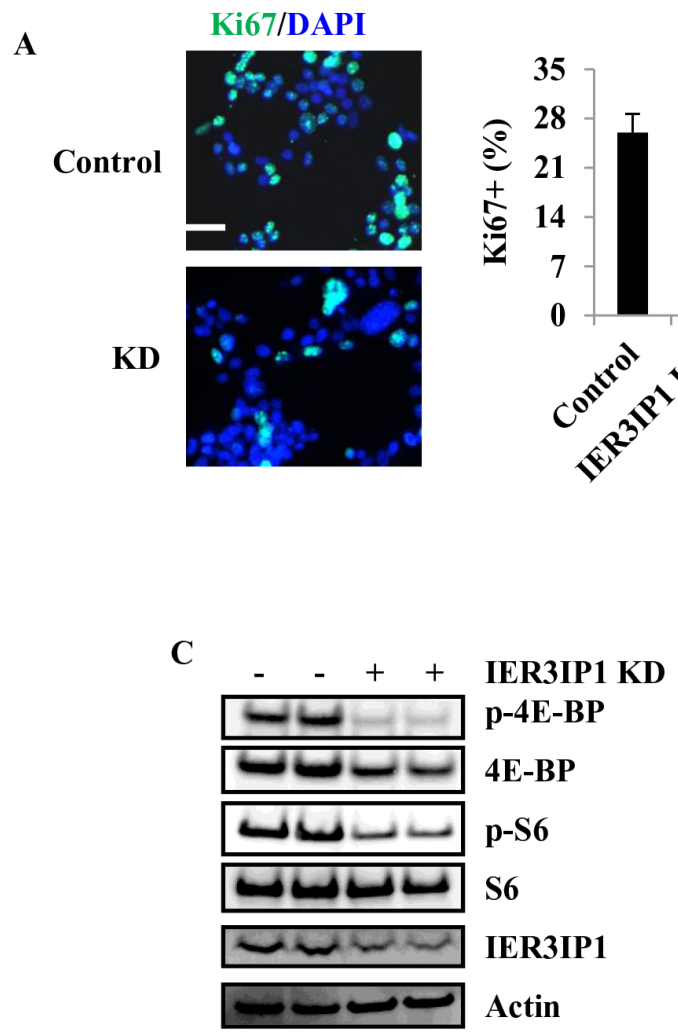

p-AKT, p-4E-BP and p-S6, which was consistent with the reduction in cell proliferation in IER3IP1 KD MIN6 cells. In addition, the reduction in p-Erk may be related to the decrease in cell proliferation rather than Bim regulation. Furthermore, PERK-deficient mice exhibit severe defects in fetal/neonatal $\beta$ cell proliferation and differentiation, resulting in low $\beta$-cell mass [29]. Since IER3IP1 KD induced a decrease in PERK, the reduction of PERK may also contribute to the decrease in cell proliferation in MIN6 cells.

In conclusion, we have shown that IER3IP1 suppression leads to apoptotic cell death. Bim upregulation and the reduction of $\mathrm{Bcl}-\mathrm{xL}$ play critical roles in mediating apoptosis in MIN6 cells. Moreover, FoxO1, Fox3a, Erk and Chop are not involved in Bim upregulation in IER3IP1-suppressed cells, and IER3IP1 is required for UPR activation and cell proliferation. These results suggest Bim, Bcl-xL and UPR may be the targets for therapeutic interventions in diabetes associated with mutations in IER3IP1. IER3IP1 may be a potential target for pancreatic cancer treatment.
B

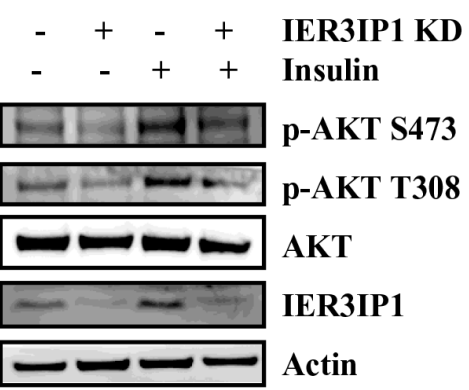

D

IER3IP1 suppression<smiles>CCCCC</smiles>

$\uparrow$ cell death cell proliferation $\downarrow$

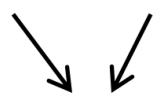

MIN6 cells defect in survival and proliferation

Figure 7: IER3IP1 suppression decreases MIN6 cells proliferation. (A) Ki-67 staining of MIN6 cells. 2 days after IER3IP1 KD in MIN6 cells, Ki-67 staining for proliferation cell nuclei was performed in MIN6 cells. The Ki-67 staining was shown in the left. Scale bar, $20 \mu \mathrm{m}$. Quantitative Ki-67 staining data were shown in the right. Values are means $\pm \mathrm{SEM}, * \mathrm{P}<0.05$ compared to control cells. (B+C) Protein levels of phosphorylation of AKT, Erk, 4E-BP and S6. 4 days after IER3IP1 KD in MIN6 cells, phosphorylation of AKT (B), 4EBP and S6 (C) were determined by Western blot. The loaded proteins in the two "- or +" lines were the same but from the different plates. (D) Model presents how IER3IP1 suppression affects MIN6 cells. IER3IP1 suppression induces an increase in cell death and a decrease cell proliferation in MIN6 cells. 


\section{MATERIALS AND METHODS}

\section{MIN6 cell culture}

Mouse MIN6 insulinoma cells were cultured in Dulbecco's modified Eagle's medium supplemented with $15 \%$ FBS, antibiotics (100 units/ml penicillin and $100 \mu \mathrm{g} /$ $\mathrm{ml}$ streptomycin), $1 \mathrm{~mm}$ sodium pyruvate, $10 \mathrm{~mm}$ HEPES, and $50 \mu \mathrm{m} \beta$-mercaptoethanol. The cells were maintained at $37{ }^{\circ} \mathrm{C}$ in an atmosphere of $5 \% \mathrm{CO}_{2}$ and $100 \%$ humidity.

\section{Quantitation of cell death}

Cell death was quantified by propidium iodide (PI) staining followed by flow cytometric analyses (FACS) using a FACS Caliber (BD Bioscience) and FlowJo software [35]. Propidium iodide intercalates into doublestranded nucleic acids. PI is excluded by viable cells but can penetrate membranes of dying or dead cells. 20 $\mu \mathrm{M}$ Z-VAD-FMK (carbobenzoxy-valyl-alanyl-aspartyl[Omethyl]-fluoromethylketone) was added to the medium 2 hours prior to treatment of MIN6 cells by IER3IP1 shRNA lentivirus. Z-VAD was added to the cells on day1 and day3.

\section{Ki67 staining}

Cell proliferation was assessed by Ki67 staining. 3 days after IER3IP1 KD, MIN6 cells were stained with Ki67-FITC (652409, BioLegend) according to the manufacturer's instructions. In brief, cells were washed with PBS, fixed in $70 \%$ ethanol at $20^{\circ} \mathrm{C}$ for 1 hour, then washed 3 times with BioLegend Cell Staining Buffer and resuspend the cells at the concentration of 0.5-10 x 10\% ml. Cell suspension was mixed with FITC-conjugated $\mathrm{Ki}-67$ antibody and incubated at room temperature in the dark for 30 minutes. Then cells were washed 2 times with BioLegend Cell Staining and resuspend in $0.5 \mathrm{ml}$ cell staining buffer for flow cytometric analysis.

\section{Quantification of mRNA levels}

RNA isolation, first strand cDNA synthesis, and TaqMan gene expression assays were performed as previously described [16]. RNA levels were normalized to the amount of actin mRNA in the same sample. Applied Biosystems (Foster City, CA) TaqMan assay numbers were: Mouse actin B, 4352933; IER3IP1, Mm01263577 g1; Bim, Mm00437796_m1 and Puma, Mm00519268_m1.

\section{Retrovirus infection}

Human Bcl-xL was cloned into the retroviral expression vector MSCV-IRES-GFP (pMIG) (Addgene). The production of amphotropic retroviruses using the 293GPG packing cell line was performed as described previously [16]. Retroviral plasmids were transfected using FuGene6 (Roche) according to the manufacturer's protocols. Retroviral transduction of each indicated BCL2 family protein was confirmed by western blotting. MIN6 cells were infected with these retroviralvectors at multiplicity of infection (MOI) of 10. Three days after infection with Bcl-xL retrovirus, MIN6 cells were lysed and probed with antibody for immunoblot analysis.

\section{Lentivirus-mediated shRNA expression}

The pLKO.1-puro lentiviral vector was generously provided by Dr. Sheila Stewart of Washington University Medical School (St. Louis, MO). Potential shRNA targets in the murine IER3IP1 mRNA (thermal Scientific) were identified by quantitative reverse transcriptase PCR (QRTPCR). Recombinant lentiviral particles were prepared by transfecting HEK $293 \mathrm{~T}$ cells with the appropriate pLKO.1-puro plasmid plus pHR'CVM8.2 delta $\mathrm{R}$ and pCMV-VSV-G plasmids. Lentivirus was added to the medium on day 1.

\section{Western blot}

MIN6 cells were lysed in cell lysis buffer containing $1 \%$ Triton X-100, $1 \mathrm{~mm}$ EDTA, $1 \mathrm{~mm}$ EGTA, $10 \mathrm{~mm}$ dithiothreitol, $1 \mathrm{~mm} \mathrm{Na} \mathrm{VO}_{4}$, and complete protease inhibitor mixture. Equal amounts of protein were resolved by $10 \%$ or $4-12 \%$ NuPAGE (Invitrogen) gels, transferred onto PVDF membrane (Millipore), and blots were probed with antibodies against IER3IP1 (sc-84849; Santa Cruz), Puma (7467; Cell Signaling Technologies), Bim (202000; Calbiochem), cleaved caspase-3 (9661; Cell Signaling Technologies), p-FoxO1/Fox3a (9461; Cell signaling), FoxO1 (2880; Cell signaling), Fox3a (2497; Cell signaling), $\beta$-actin (A-2066; Sigma), Bax (6A7) (2281MC-100; Trevigen), Bax (N20) (sc-493, Santa Cruz), Bak (upstate, 06536), p-IRE1 $\alpha$ (NB100-2323; Novus), IRE1 $\alpha$ (3294; Cell signaling), p-PERK (3179, Cell signaling), PERK (sc-9477; Santa Cruz), ATF4 (ARP37017_P050, Aviva systems biology); p-eIF2a (9721, Cell signaling), eIF2a (9722; Cell signaling), and Chop (2895; Cell signaling). To detect BAX activation, immunoprecipitation (IP) was performed using $1 \%$ CHAPS buffer ( $1 \%$ CHAPS, $142.5 \mathrm{mM} \mathrm{KCl}, 2 \mathrm{mM} \mathrm{CaCl}, 20 \mathrm{mM}$ Tris-Cl, $\mathrm{pH}$ 7.4). Anti-6A7 IP was performed using 1\% CHAPS buffer. Antibody detection was accomplished using enhanced chemiluminescence (PerkinElmer) and LAS-3000 Imaging system (FUJIFILM).

\section{Flow cytometric analysis of mitochondrial membrane potential}

Mitochondrial membrane potential was assessed by TMRE (tetramethylrhodamine, ethyl ester) staining followed by flow cytometric analysis [36]. TMRE enters cells and reversibly accumulates in the highly negatively charged mitochondrial matrix according to the Nernst equation, allowing the potential to be measured. 


\section{Immunofluorescence analysis of cytochrome c}

After 3 days treatment with lentiviral control or IER3IP1 shRNA, MIN6 cells were fixed for $15 \mathrm{~min}$ in $4 \%$ paraformaldehyde, permeabilised with $0.5 \%$ Triton $\mathrm{X}-100 / \mathrm{PBS}$ for $5 \mathrm{~min}$ and then incubated for $1 \mathrm{~h}$ in a $5 \%$ BSA/PBS blocking solution. Then cells were incubated for overnight at $4{ }^{\circ} \mathrm{C}$ with a mouse monoclonal anticytochrome c IgG (Pharmingen) followed by exposure to a goat anti-mouse Alexa488-conjugated secondary antibody (invitrogen). Images were obtained using an Evos microscope (Advanced microscope group). The release of cytochrome $\mathrm{C}$ was measured by ELISA kit (Abcam).

\section{TUNEL staining}

The apoptotic cell death was determined by the terminal deoxynucleotidyltransferase-mediated dUTP nick end labeling (TUNEL) labeling (Promega Corp) according to the manufacturer's instructions.

\section{Statistical analysis}

The 2-tailed unpaired Student's t test was used to assess the statistical significance of differences between 2 sets of data. Differences were considered significant when $\mathrm{P}<0.05$. In all experiments, the number of asterisks is used to designate the following levels of statistical significance: $* * * \mathrm{P}<0.001,{ }^{* *} \mathrm{P}<0.01, * \mathrm{P}<0.05$ compared with control group. \#\#\# $\mathrm{P}<0.001$, \#\#P $<0.01$, $\# \mathrm{P}<0.05$ compared with IER3IP1 KD group. Results are presented as mean $\pm \mathrm{SEM}$. The data are representative experiments from another two with very similar results.

\section{Author contributions}

D.R. designed research; D.R. and J.S. performed research; D.R. and J.S. analyzed data; and D.R. wrote the paper.

\section{ACKNOWLEDGMENTS}

The authors thank Dr. K. Polonsky for insightful suggestions, advice and discussions.

This study was supported by P30-DK020595 (The University of Chicago and Diabetes Research and Training Center).

\section{CONFLICTS OF INTEREST}

No potential conflicts of interest relevant to this study were reported.

\section{REFERENCES}

1. Poulton CJ, Schot R, Kia SK, Jones M, Verheijen FW, Venselaar H, de Wit MC, de Graaff E, Bertoli-Avella AM,
Mancini GM. Microcephaly with simplified gyration, epilepsy, and infantile diabetes linked to inappropriate apoptosis of neural progenitors. Am J Hum Genet. 2011; 89:265-276.

2. Abdel-Salam GM, Schaffer AE, Zaki MS, Dixon-Salazar T, Mostafa IS, Afifi HH, Gleeson JG. A homozygous IER3IP1 mutation causes microcephaly with simplified gyral pattern, epilepsy, and permanent neonatal diabetes syndrome (MEDS). Am J Med Genet A. 2012; 158A:2788-2796.

3. Shalev SA, Tenenbaum-Rakover Y, Horovitz Y, Paz VP, Ye H, Carmody D, Highland HM, Boerwinkle E, Hanis CL, Muzny DM, Gibbs RA, Bell GI, Philipson LH, Greeley SA. Microcephaly, epilepsy, and neonatal diabetes due to compound heterozygous mutations in IER3IP1: insights into the natural history of a rare disorder. Pediatr Diabetes. 2014; 15:252-256.

4. Leonardi O, Mints G, Hussain MA. Beta-cell apoptosis in the pathogenesis of human type 2 diabetes mellitus. Eur J Endocrinol. 2003; 149:99-102.

5. Ron D, Walter P. Signal integration in the endoplasmic reticulum unfolded protein response. Nat Rev Mol Cell Biol. 2007; 8:519-529.

6. Fonseca SG, Gromada J, Urano F. Endoplasmic reticulum stress and pancreatic beta-cell death. Trends Endocrinol Metab. 2011; 22:266-274.

7. Lee DH, Park T, Kim HW. Induction of apoptosis by disturbing mitochondrial-membrane potential and cleaving PARP in Jurkat $\mathrm{T}$ cells through treatment with acetoxyscirpenol mycotoxins. Biol Pharm Bull. 2006; 29:648-654.

8. Kim H, Tu HC, Ren D, Takeuchi O, Jeffers JR, Zambetti GP, Hsieh JJ, Cheng EH. Stepwise activation of BAX and BAK by tBID, BIM, and PUMA initiates mitochondrial apoptosis. Mol Cell. 2009; 36:487-499.

9. Fingar DC, Richardson CJ, Tee AR, Cheatham L, Tsou C, Blenis J. mTOR controls cell cycle progression through its cell growth effectors S6K1 and 4E-BP1/eukaryotic translation initiation factor 4E. Mol Cell Biol.. 2004; 24:200-216.

10. Morita M, Gravel SP, Hulea L, Larsson O, Pollak M, St-Pierre J, Topisirovic I. mTOR coordinates protein synthesis, mitochondrial activity and proliferation. Cell Cycle. 2015; 14:473-480.

11. Putcha GV, Le S, Frank S, Besirli CG, Clark K, Chu B, Alix S, Youle RJ, LaMarche A, Maroney AC, Johnson EM Jr. JNK-mediated BIM phosphorylation potentiates BAXdependent apoptosis. Neuron. 2003; 38:899-914.

12. Ludwinski MW, Sun J, Hilliard B, Gong S, Xue F, Carmody RJ, DeVirgiliis J, Chen YH. Critical roles of Bim in T cell activation and $\mathrm{T}$ cell-mediated autoimmune inflammation in mice. J Clin Invest. 2009; 119:1706-1713.

13. Craxton A, Draves KE, Clark EA. Bim regulates BCRinduced entry of B cells into the cell cycle. Eur J Immunol. 2007; 37:2715-2722. 
14. Erlacher M, Labi V, Manzl C, Bock G, Tzankov A, Hacker G, Michalak E, Strasser A, Villunger A. Puma cooperates with Bim, the rate-limiting $\mathrm{BH} 3$-only protein in cell death during lymphocyte development, in apoptosis induction. J Exp Med. 2006; 203:2939-2951.

15. Kirschnek S, Ying S, Fischer SF, Hacker H, Villunger A, Hochrein H, Hacker G. Phagocytosis-induced apoptosis in macrophages is mediated by up-regulation and activation of the Bcl-2 homology domain 3-only protein Bim. J Immunol. 2005; 174:671-679.

16. Sun J, Mao LQ, Polonsky KS, Ren DC. Pancreatic $\beta$-cell death due to $\mathrm{Pdx}-1$ deficiency requires multi-BH domain protein Bax but not Bak. J Biol Chem. 2016; 291:13529-13534.

17. Ren D, Sun J, Wang C, Ye H, Mao L, Cheng EH, Bell GI, Polonsky KS. Role of BH3-only molecules Bim and Puma in beta-cell death in Pdx1 deficiency. Diabetes. 2014; 63:2744-2750.

18. Ren D, Tu HC, Kim H, Wang GX, Bean GR, Takeuchi O, Jeffers JR, Zambetti GP, Hsieh JJ, Cheng EH. BID, BIM, and PUMA are essential for activation of the BAXand BAK-dependent cell death program. Science. 2010; 330:1390-1393.

19. Campbell PD, Weinberg A, Chee J, Mariana L, Ayala R, Hawthorne WJ, O'Connell PJ, Loudovaris T, Cowley MJ, Kay TW, Grey ST, Thomas HE. Expression of pro- and antiapoptotic molecules of the Bcl-2 family in human islets postisolation. Cell Transplant. 2012; 21:49-60.

20. Santin I, Moore F, Grieco FA, Marchetti P, Brancolini C, Eizirik DL. USP18 is a key regulator of the interferondriven gene network modulating pancreatic beta cell inflammation and apoptosis. Cell Death Dis. 2012; 3:e419.

21. Nogueira TC, Paula FM, Villate O, Colli ML, Moura RF, Cunha DA, Marselli L, Marchetti P, Cnop M, Julier C, Eizirik DL. GLIS3, a susceptibility gene for type 1 and type 2 diabetes, modulates pancreatic beta cell apoptosis via regulation of a splice variant of the $\mathrm{BH} 3$-only protein Bim. PLoS Genet. 2013; 9:e1003532.

22. Barreyro FJ, Kobayashi S, Bronk SF, Werneburg NW, Malhi H, Gores GJ. Transcriptional regulation of Bim by FoxO3A mediates hepatocyte lipoapoptosis. J Biol Chem. 2007; 282:27141-27154.

23. Urbich C, Knau A, Fichtlscherer S, Walter DH, Bruhl T, Potente M, Hofmann WK, de Vos S, Zeiher AM, Dimmeler $\mathrm{S}$. FOXO-dependent expression of the proapoptotic protein Bim: pivotal role for apoptosis signaling in endothelial progenitor cells. FASEB J. 2005; 19:974-976.

24. Ley R, Ewings KE, Hadfield K, Cook SJ. Regulatory phosphorylation of Bim: sorting out the ERK from the JNK. Cell Death Differ. 2005; 12:1008-1014.
25. Ewings KE, Wiggins CM, Cook SJ. Bim and the prosurvival Bcl-2 proteins: opposites attract, ERK repels. Cell Cycle. 2007; 6:2236-2240.

26. Doonan F, Donovan M, Gomez-Vicente V, Bouillet P, Cotter TG. Bim expression indicates the pathway to retinal cell death in development and degeneration. J Neurosci. 2007; 27:10887-10894.

27. Kim I, Xu W, Reed JC. Cell death and endoplasmic reticulum stress: disease relevance and therapeutic opportunities. Nat Rev Drug Discov. 2008; 7:1013-1030.

28. Delepine M, Nicolino M, Barrett T, Golamaully M, Lathrop GM, Julier C. EIF2AK3, encoding translation initiation factor 2-alpha kinase 3, is mutated in patients with WolcottRallison syndrome. Nat Genet. 2000; 25:406-409.

29. Zhang W, Feng D, Li Y, Iida K, McGrath B, Cavener DR. PERK EIF2AK3 control of pancreatic beta cell differentiation and proliferation is required for postnatal glucose homeostasis. Cell Metab. 2006; 4:491-497.

30. Lee AH, Heidtman K, Hotamisligil GS, Glimcher LH. Dual and opposing roles of the unfolded protein response regulated by IRE1alpha and XBP1 in proinsulin processing and insulin secretion. Proc Natl Acad Sci U S A. 2011; 108:8885-8890.

31. Hiramatsu N, Chiang WC, Kurt TD, Sigurdson CJ, Lin JH. Multiple mechanisms of unfolded protein response-induced cell death. Am J Pathol. 2015; 185:1800-1808.

32. Orrenius S, Zhivotovsky B, Nicotera P. Regulation of cell death: the calcium-apoptosis link. Nat Rev Mol Cell Biol. 2003; 4:552-565.

33. Balcazar Morales N, Aguilar de Plata C. Role of AKT/ mTORC1 pathway in pancreatic beta-cell proliferation. Colomb Med (Cali). 2012; 43:235-243.

34. Dickson LM, Rhodes CJ. Pancreatic beta-cell growth and survival in the onset of type 2 diabetes: a role for protein kinase B in the Akt? Am J Physiol Endocrinol Metab. 2004; 287:E192-198.

35. Boyd V, Cholewa OM, Papas KK. Limitations in the use of fluorescein diacetate/propidium iodide (FDA/PI) and cell permeable nucleic acid stains for viability measurements of isolated islets of langerhans. Curr Trends Biotechnol Pharm. $2008 ; 2: 66-84$.

36. Jayaraman S. Flow cytometric determination of mitochondrial membrane potential changes during apoptosis of T lymphocytic and pancreatic beta cell lines: comparison of tetramethylrhodamineethylester (TMRE), chloromethylX-rosamine (H2-CMX-Ros) and MitoTracker Red 580 (MTR580). J Immunol Methods. 2005; 306:68-79. 